\title{
EMANZIPIERTES KÜNSTLERTUM AM BEISPIEL VON WERKEN ELFRIEDE JELINEKS
}

Elfriede Jelinek und ihr Schaffen haben stets viel Interesse geweckt. Ihre Literatur wurde nicht nur gelesen, weil die Autorin schon immer zu einer bestimmten Elite unter den Schriftstellern gehörte, man las sie, um ein Thema für Kritik und Kommentare zu haben. „Wer vor Jelineks Texten erschrickt, erschrickt vor sich selbst, vor seinen Rachefantasien, seiner Wut auf den früheren oder jetzigen Partner, die einengende Mutter und anderes. "' Der Nobelpreis hat nun Jelineks Freunde und Feinde noch einmal zu Wort kommen lassen. Sie erhielten noch einmal die Chance, in Zeitschriften und Zeitungen sowie in der Fachpresse die eigene Meinung darzustellen, was die meisten auch taten. „Öffentlich erscheint sie“, heißt es zum Beispiel, „distanziert und distanzierend, sie mușs gar nichts Provokantes mehr äuBern, um zum Beispiel bei Journalisten - und damit Lesern und Nicht-einmalLesern ihrer Texte aggressive Phantasien zu evozieren. “²

\section{Individualismus (Einzelpersönlichkeit) als Protest gegen Vermassung}

\section{„Klavierspielerin“}

„Wenn etwas besonders unverwechselbar ist, dann nennt man es Erika.“3 Der Individualismus von Erika spiegelt sich in den Verhältnissen in der geraden Linie Mutter - Tochter. Das erwachsene Kind wird als solches nie betrachtet, wird in den Augen der Mutter zu einer Einzigartigkeit. Erika wiederholt keine allgemein be-

\footnotetext{
${ }^{1}$ Elfriede Gerstl: Schrillers weiß ich heute nicht anzubieten. Die Frau als Wutableiter, S. 1.

${ }^{2}$ Elfriede Gerstl: Ebd., S. 1.

${ }^{3}$ Elfriede Jelinek: Die Klavierspielerin, Roman, Reinbek 1986, S. 14.
} 
kannten Muster der Erziehung und des Erwachsenseins. Das Mädchen, schon wieder wie eine Frau, muss sich deutlich von allen anderen unterscheiden. Die Mutter als Herrin über Schicksal und Leben der eigenen Tochter wählt für sie die beste Zukunft und erfüllt dabei wahrscheinlich eigene unerfüllte Wünsche und Träume. Vermutet werden kann, dass sie große Angst vor der eigenen Tochter als Partnerin hat. Mit allen möglichen Mitteln möchte sie die Tochter als ewiges Kind beibehalten. Die Entwicklung, die Schaffung des eigenen Ichs wirken sehr deprimierend auf die Mutter. Sie fühlt sich nicht mehr imstande, über das Leben der Tochter zu entscheiden, über sie herrschen zu können. „Für Erika wählt die Mutter früh einen in irgendeiner Form künstlerischen Beruf, damit sich aus der mühevoll errungenen Feinheit Geld herauspressen läßt, während die Durchschnittsmenschen bewundernd um die Künstlerin herumstehen, applaudieren. " ${ }^{4}$ Der Ehrgeiz der Mutter muss von der Tochter verwirklicht werden, ohne dass diese jemals danach gefragt wurde. Einerseits besteht die Mutter darauf, aus dem eigenen Kind eine Individualistin zu machen, andererseits hat sie furchtbare Angst vor jeder selbständigen Entscheidung Erikas. Der Zwangsindividualismus teilt sich in dieser Situation durch zwei, die beiden Frauen bilden ein untrennbares Tandem, das man nicht separat untersuchen kann. Die Einzigartigkeit der Tochter wird, so unglaublich das klingt, von der Mutter bestimmt und geschaffen, der Individualismus der Tochter hängt von der Mutter ab. Erika selbst hat nur der Mutter zu folgen und ihre Befehle zu erfüllen. Ihr Denken wird ausgeschaltet, ersetzt durch die Herrschaft der Mutter. „Auch Erika hat sich den Trivialmythos der Einzigartigkeit und herausragenden ,Einzelpersönlichkeit' zu Eigen gemacht. Sie tritt entschieden, gegen Vermassung' auf und bezeichnet sich als Individualistin." 5 Durch die seitens der Mutter ergriffenen Erziehungsmaßnahmen, wenn man in diesem Alter eines Kindes überhaupt noch über Erziehung reden kann, wird Erika zu einem Menschen, der das Leben und die Menschen bewusst nicht genießen kann. Man kann in dieser Analyse noch tiefer gehen und feststellen, dass alle Befürchtungen der Mutter zur vollständigen Isolation des Mädchens führen und das Missvertrauen ihr gegenüber noch steigern. Bedeutet eine Einzelgängerin genau das Gleiche wie eine Individualistin? Die Mutter sieht darin wahrscheinlich keinen Unterschied, für die Außenwelt gilt Erika kaum als eine Einzelpersönlichkeit im positiven Sinne, eher wird sie als Outsiderin, eine durch die Mutter dominierte, unselbstständige kleine Frau betrachtet. Kann das Streben der Mutter als ein Protest gegen die Vermassung verstanden werden? Wenn man die Zukunft einer Klaviererspielerin betrachtet, mit allen erhofften Privilegien, Erfolgen, Preisen, ist das bestimmt ein Protest und ein Weg, wie man zu einem Individuum wird. Die Realität zeigt jedoch, dass dieser Weg nicht immer gerade verläuft, dass man dabei auf das alltägliche Leben nicht verzichten kann. Eine talentierte und weltberühmte Pianistin wird man nicht automatisch. Die Erwartungen der Mutter

\footnotetext{
${ }^{4}$ Elfriede Jelinek: Die Klavierspielerin, S. 25.

${ }^{5}$ Merlies Janz: Elfriede Jelinek, 1995, S. 73.
} 
werden durch eine Stelle als Lehrerin in der Musikschule ersetzt. Ob das immer noch der Protest sein kann? Solange man auf etwas Besonderes hofft und darauf eingestellt ist, in diesem Falle auf eine blühende Karriere als Klavierspielerin, kann man die eigene Einzigartigkeit als eine Mission betrachten. Durch den Status als Lehrerin indes werden die Träume drastisch entzaubert und verlieren an Farbigkeit und dadurch auch an Bedeutung.

\section{Entzug der eigenen Identität. Männliche Weiblichkeit. Rollenübergabe der Mutter und der Tochter.}

\section{„Die Klavierspielerin“}

Der Wille der Mutter scheint für beide Frauen zu gelten. Erika darf keine eigene Meinung haben, sie wird dann automatisch bestraft, und bevor sie es wagt, selbständig zu entscheiden, sieht sie die Reaktion der unzufriedenen und wütenden Mutter voraus. Der Tochter werden verschiedene Rollen zugeteilt. Nach dem Verlust des Vaters wird sie zu ihrem eigenen Vater, zu dem Mann für ihre Mutter, zum Betreuer des Hauses. Die Übernahme vieler Pflichten und vor allem die psychische Anpassung an die Erwartungen der Mutter zerstören das eigene Ich der jungen Frau. „Erika ist für die Mutter Mann-Ersatz; Mutter und Tochter schlafen im Ehebett. Aber auch umgekehrt ersetzt die Mutter für Erika den Mann und macht ihr die Beziehung zu einem Mann auf vertrackte Weise unmöglich.“6 „Nie könnte sie sich zu einem Mann unterordnen, nachdem sie sich so viele Jahre der Mutter untergeordnet hat. Sie hat noch ein Mütterlein und braucht daher keinen Mann zu frein. “7 In der Relation beider Frauen fehlt ein Mann. Diese Lücke kann auf Dauer nicht vernachlässigt werden. Eine entscheidende Rolle spielt hier auch die Eifersucht der Mutter, ihr eigenes Kind zu verlieren, etwa durch das Erscheinen eines neuen Mannes im Haus, der nur für die Tochter da sein müsste. Sehr wichtig schein hier auch die Sexualität zu sein. Die selbstverständlichen Unterschiede zwischen der Mutter und der Tochter, wie Alter, Ausbildung, Erfahrung, Erwartungen, Selbstentwicklung, haben keinen gemeinsamen Punkt. Einen Kompromiss zu finden scheint unmöglich zu sein. Die Mutter löst dieses Problem - wenn auch unbewusst - automatisch, indem sie über den Entzug von Freiheit und dem Selbstbestimmungsrecht der Tochter alles alleine regelt und bestimmt. In ihren Augen muss Erika die Entscheidungen und Befehle der eigenen Mutter als artige Tochter akzeptieren und sie auch ausführen. Sexualität ist in diesem Haus ein Tabuthema, was zu vielen Störungen in den Relationen Erika - Mann führt. Als Lehrerin hat sie dann die Möglichkeit der eigenen Herrschaft über ihre Schüler, die sie auch nutzt. Der Mangel an Kenntnis-

\footnotetext{
${ }^{6}$ Ebd., S. 71.

${ }^{7}$ Elfriede Jelinek: Die Klavierspielerin, a.a. O., S. 15.
} 
sen über den eigenen Körper und über ihre Weiblichkeit macht aus Erika ein großes Kind mit den natürlichen Bedürfnissen einer erwachsenen Frau. Die Mutter muss mit Blindheit geschlagen sein, da sie die hierin verborgene große Gefahr nicht wahrnimmt. Erika mit allen ihren Mängeln, ihrem krankhaften Selbstbewusstsein und ihrer Unsicherheit kann nur zum Opfer werden. Die heimliche Analyse von pornographischen Zeitschriften, Selbsterkenntnis des eigenen Körpers, tragen dazu bei, dass Erika mit Aggressivität wirkt. Diese Aufgeregtheit und der Zorn sind aber sehr oberflächlich. Auf diese Art und Weise schont sie sich selbst und sucht nach einem neuen Weg, einem Weg, der nur ihr gehören wird, auf dem es keinen Platz für die Mutter gibt. Ein solches Denken führt jedoch sofort zu Gewissensbissen, verdankt sie doch alles der Mutter; sie wohnt mit ihr zusammen, die Mutter verfolgt sie, wartet auf sie, alles dreht sich um die Mutter. Klavierspielerin zu sein sollte ihre Weiblichkeit ersetzen und dazu führen, sie ausschließlich als Künstlerin zu betrachten. Die Mutter hat bewusst ihr eigenes Kind unglücklich machen wollen, vielleicht aus purer Angst, dass sie das Leben der eigenen Eltern wiederholt, vielleicht weil sie eine Ausnahmetochter haben wollte. Im Endeffekt betrachtet sich Erika selbst als „es“. Weder Frau noch Künstlerin. Die männlichen Eigenschaften, die ihr aufgedrängt wurden, scheinen sich in den Beziehungen zu Männern zu melden. Erika ist nicht in der Lage, offen über eigene Gefühle zu reden, ein eigenes Leben getrennt von der Mutter beginnen zu können, sie ist zu unerfahren und unaufgeklärt, um die eigene Sexualität in eine gute Richtung zu lenken. Ein Kompromiss bleibt bei all dem ausgeschlossen. „Der Frust und Zwang, der Fräulein Kohut zur Reglosigkeit fesselt, knebelt, kettet, hat Folgen für ihr Sexualverhalten. Das ist wohl psychologisch richtig. Erika Kohut wird von einer Voyeurin zur Masochistin. Ihre Phantasien sind horrend. ${ }^{\text {(8 }}$ Die Provokation des Schülers Klemmer kann nur auf eine Art und Weise enden. Im Spielen mit seinen Gefühlen spiegelt sich das zu Hause erfahrene Modell wider. Nach der langen Unterordnung unter die Mutter will swie Revanche üben und Herrschaft über Klammer erlangen. Wird Klemmer zum Opfer? Eigentlich die einzige, die verliert ist wiederum Erika. Die Beziehung, die sie sich als Liebe erklärt, endet mit der Vergewaltigung der Frau. So eine Reaktion des Jungen war zu erwarten, zuerst ihre sexuellen Spielen, die von Erika unterbrochen waren, dann der Brief, in dem sie alle ihren Erwartungen beschrieben hat, und sie Klemmer vorlesen ließ.

\section{„Lust"“}

Ein ganz ähnliches Bild von Provokation und Gegenreaktion finden wir in dem Roman Lust. Die dort dargestellte Szene der Ehe zwischen Hermann und seiner Frau ist zugleich ein Beispiel für Unterordnung und Unselbstständigkeit. „Der Leser hat nach 225 Seiten Lektüre Augenblicke des Ausruhens, der Entlastung nötig; vom

\footnotetext{
${ }^{8}$ Reinhard Beuth: Treffsicher im Giftspritzen, in: Elfriede Jelinek: Dossier, S. 202.
} 
immer wieder, in einem unerbitterlichen Rhythmus heraufgereizten Gefühl des Widerwillens, des Ekels, des Grauens (...).“9 Die Unterordnung der Frau ist verknüpft mit den den Ehepartnern zugeteilten typischen Rollen. Jelinek geht es hier nicht nur um eine auf Haushalt oder Arbeit bezogene Teilung, sondern auch um die psychische Drangsalierung der Frau. Radikale Destruktion, Schrecken, Angst - mit diesen Mitteln führt Jelinek zu einer Familientragödie hin, die jahrelang dauert und gegen die niemand etwas unternimmt. Zwei Opfer sind in diesem Roman zu nennen. Als sehr markante Figur erscheint die Ehefrau - Gerti, die von ihrem Mann ausgenutzt wird. Sein sexueller Drang und seine Potenz zerstören die Frau. „Der Körper der Frau als Ware - aber die unendliche Sexualität ist jenseits aller realistischen Annnahmen angesiedelt: Von Trieb und Perversion läßt sich kaum mehr sprechen, nicht mehr von menschlicher Natur. “10 Die monotone Situation zu Hause, der ständige Druck des Mannes führen Gerti zu einem völligen Verlust der eigenen Identität. Die Weiblichkeit dieser Frau wurde längst getötet, die Familienhölle scheint ihr ganzes Leben dominiert und beeinflusst zu haben, wobei die Dauer dieser Tragödie sie offenbar verzweifelt und hoffnungslos gemacht hat. Die Frau träumt von Gefühlen, von Stille, Genuss, Zusammensein. Alles, was sie bekommt, ist Drang, Stress, Gefühllosigkeit und Kälte. Einmal versucht sie sich von dieser Last zu befreien, als sie in einem jungen Jurastudenten eine Chance und eine Hoffnung erblickt. Ihre Hoffnung, wonach dieser Student die Situation retten und aus ihr doch noch eine richtige Frau machen soll, wird jedoch enttäuscht. „Gefühle sind die Nahrung der Armen, also der Frauen: Michael ist wie ihr Mann, erkennt im Spiegel sich als seinen eigenen Gott.“11 Die Erwartungen und die Naivität der Frau werden von Michael brutal ausgenutzt. Die Vergewaltigung von Gerti stiehlt ihre letzten Wünsche, Träume, Gefühle, tötet ein weiteres Mal die übriggebliebenen weiblichen Instinkte. Verschlimmert wird die Situation noch durch ihren Mann, der im Anschluss seine Frau „demonstrativ vor Michael sexuell in Besitz nimmt" ${ }^{12}$ „Über dieser Welt aus Geld, Gewalt und Gier thront der sprechend Hermann genannte Haupttäter, ein patriarchal-kapitalistisches Doppelmonster aus dem sozialkritischen Klischeearsenal. “'13 Dieser Mann - als brutale, gefühllose Figur dargestellt - wird in gewissem Sinne zum Opfer seines eigenen Ichs. Geld, Arbeit, Dominanz, Brutalität und Sex machen aus ihm eine dominierende Person. Sowohl zu Hause als auch in der Fabrik spielt er stets die erste Geige, trifft selbst alle Entscheidungen, trägt die Verantwortung und leitet ein großes Unternehmen. Sein Drang nach Sex und Befriedigung soll emotionale Unzulänglichkeiten ersetzen oder sie vertuschen. Das wehrlose Opfer, zu dem er seine eigene Frau macht, meldet sich nie zu Wort, es wird auch nirgendwo Hilfe suchen. Das weißt er zu gut, um Angst zu bekommen,

\footnotetext{
${ }^{9}$ Klaus-Peter Philippi, in: Elfriede Jelinek: Dossier, S. 234.

${ }^{10}$ Ebd., S. 235.

${ }^{11}$ Ebd., S. 236.

12 Ebd., S. 236.

${ }^{13}$ Andreas Isenschmid, in: Elfriede Jelinek: Dossier, S. 240.
} 
allein zu bleiben. Die demütigende Praxis und der inszenierte Verlauf jedes Tages zu Hause sind Ritual. Aus dem Körper von Gerti macht er ein Objekt der eigenen Lust.

\title{
Musik als Ersatz des Trivialmythos - der Liebe. Verzicht auf die bewusste Anwesenheit des Mannes im Leben einer Frau. Verzicht auf emotionale Entwicklung eigenen Ichs
}

\author{
„Insekt in Bernstein ${ }^{\text {(14 }}$
}

Die Musikübungen diktieren den Verlauf von Erikas Kindheit. Überwacht und kontrolliert wird sie Tag und Nacht von der Mutter. "Erst in der Pubertät wird das Üben an den Instrumenten als Strategie der Mutter deutlich: verschärfte Einsperrung, keine Zeit für andere Menschen. “15 Alle Wünsche des Mädchens werden von der Mutter niedergekämpft. Ziel des Übens ist das Erreichen der Weltspitze - die von der Mutter ausgewählten Mittel zum Ziel sind Fleiß, Askese, Einsamkeit. Begabung scheint nicht notwendig, sie lässt sich später entwickeln. Die Askese, die der Tochter von der Mutter abverlangt wird, führt auf Dauer zur Verkümmerung. Trotz Fortschritten am Instrument reichen diese nicht aus, um aus Erika eine Solistin zu machen. Jelinek wählt nicht zufällig das Klavierspielen als Hauptthema des Romans. „Klavier symbolisiert für die Jelinek offenbar etwas wie Fesselung." “16 „Zur Fessel kommt der Frust über die gescheiterte Kariere.“17 Der Kunstverstand der Mutter geht weit über das hinaus, was die Tochter wirklich kann. Der Mutter zufolge soll die Musik Türen öffnen und beiden eine bessere Zukunft sichern. Der Ehrgeiz der Mutter und der Gedanke, die Mutter einer weltbekannten Klavierspielerin zu sein, drängen alles andere in den Hintergrund. Die Mutter ist in die Rolle einer Klaviererinmutter hineingewachsen. „Die Tochter ist ganz eingetaucht in das Horrorkabinett der Kleinbürgerlichkeit. “18 ${ }^{18}$ Die ungelebte Jugend, die gänzlich der Musik geopfert war, lässt sich nicht nachholen. Enttäuschung über die nicht erfüllte Kariere und die Degradierung zur Musiklehrerin frustrieren sowohl Tochter als auch Mutter. Den kleinen Demütigungen der Banausen folgen jetzt die größeren, denn Erika verachtet ihre unbegabten Schüler und begibt sich weit unter ihr Niveau. " ${ }^{19}$ Das Leben zeigt die rohe Wahrheit und verifiziert die. Erwartungen der beiden Damen Kohut, nachdem es offenbar ist, dass die Chance auf eine Weltkar-

\footnotetext{
${ }^{14}$ Ria Endres: Ein musikalisches Opfer, in: Elfriede Jelinek: Dossier, S. 203.

${ }^{15}$ Ebd., S. 203.

${ }^{16}$ Reinhard Beuth: Treffsicher im Giftspritzen, in: Elfriede Jelinek: Dossier, S. 201.

${ }^{17}$ Ebd., S. 201.

${ }^{18}$ Ebd., S. 202.

${ }^{19}$ Ria Endres: Ein musikalisches Opfer, a. a. O., S. 203.
} 
riere verfallen ist. Und eben für die musikalische Aussage ihres Werkes findet die „Skandalautorin“"20 die Anerkennung der ganzen Welt, die sich u.a. in der Begründung für den Nobelpreis niederschlägt: „(...) für den musikalischen Fluss von Stimmen und Gegenstimmen in Romanen und Dramen, die mit einzigartiger sprachlicher Leidenschaft die Absurdität und zwingende Macht der sozialen Klischees enthüllen." 21

\section{Sexualität}

,Lust ${ }^{66}$

„Literatur also von Frauen für Frauen und Minderheiten, gut gemeint, aber unspannend, ästhetisch nicht ambitioniert. “22 Das Buch Lust von Jelinek beschreibt praktisch auf jeder Seite einen sexuellen Akt. Die Absicht der Autorin war es, daraus ein Pornobuch zu machen. Ob dieser Versuch geglückt ist?

„Der provozierenden und strapaziösen Unlust, die dieser Text stimuliert, stehen durchaus etliche entschädigende Lustangebote gegenüber, ohne die ihn wohl keiner zu Ende lesen würde. Dass der Wechsel und die Mischung von Unlust und Lust überhaupt für die Lektüre literarischer Texte konstitutiv sind, entspricht einer Tradition anthropologischen Wissens, an die auch Freud anknüpfte. Lust ist ohne vorangehende oder gleichzeitige Unlust nicht zu haben. Nach Freud ist die Lust der Phantasietätigkeit aus unlustvollen Mangelerfahrungen des Phantasierenden geboren." 23 Die hier beschriebenen Sexszenen enthalten viel Aggressivität, Brutalität, Obszönität. Sie schrecken nicht vor Direktheit und Offenheit. „Jelineks Texte üben eine - teilweise überzogene, typisch österreichische - Gesellschaftskritik, insgesamt betrachtet irgendwie, schon lobenswert: manchmal klingt dazu durch: aber irgendwie angestaubt, etwas totgelaufen, die Masche. Man vermittelt deutlich, dass das Provokationsmittel ihrer Texte, das man früher stets lustvoll angeprangerte oder lobte, mittlerweile konsensfähig sei.“24 „Dem Lob auf die Gesellschaftskritik in Jelineks Werk (natürlich mit Einschränkungen) folgt auf dem Fuße eine disqualifizierende Bemerkung zur literarischen Qualität der Texte. Implizit oder auch explizit werden die dabei alte Frontstellungen aktiviert, nämlich der Gegensatz von Autonomie-Ästhetik und politischer Literatur, und diese in verschiedenen Varianten serviert: Anstatt über ästhetische Verfahren spricht man konsequent nur über die Themen der Texte und über Jelineks ,Mut', ihre ,Radikalität', über ihre ungeheure

${ }^{20}$ Andrea Geier: Lob mit Fußtritten. Über den Nobelpreis für Elfriede Jelinek, in: C. H. Beck, Literaturaturkritik. Nr 11 (2004), S. 2.

${ }^{21}$ Ebd., S. 2.

${ }^{22}$ Ulrich Greiner, in: Andrea Geier: Lob mit Fußtritten, S. 5.

${ }^{23}$ Thomas Anz: Spannung durch Trennung, in: Lust und Unlust am Text, S. 3.

${ }^{24}$ Andrea Geier: Lob mit Fußtritten, a. a. O., S. 3. 
,Wut'. Mutige Kritik und Verve, das kann man loben, ohne sich etwas zu vergeben, und es fällt leicht, denn man hat es schon oft gesagt. ${ }^{\text {"25 }}$ Alles spielt sich automatisch $a b$, verläuft nach einem Schema. Es gibt dabei keinen Platz und keine Zeit für Flexibilität, Innovationen. Der Druck und die Forderungen des Mannes dominieren in diesen Szenen. „Bei Jelinek ist freilich die Stimulation von Unlust so weit getrieben, dass viele Leser und Leserinnen sich davon überfordert zeigen.“26 „Niemand lernt schließlich zu lesen ohne zu leiden. “27 Die Frau wird fast gänzlich vergessen. Eigentlich dient sie hier als ein Objekt, längst kein Subjekt mehr. „(...) einen Text, mit und in dem die Frau (als Autorin, literarische Figur oder auch Leserin) Subjekt statt Objekt sexueller Lust ist. Die Übermacht einer männlichen Sprache der Pornographie hat zum Scheitern des ursprünglichen Projekts geführt (ein pornographisches Buch für die Frauen zu schreiben) und es zu einem anderen verschoben: zum Projekt der Destruktion pornographischer Sprach- und Bildmuster und der sie begleitenden Ideologeme durch deren verfremdete Imitation und Zitation.“28 Diese Rolle wählt die Heldin aber zum Teil bewusst aus, ,konsumgeil und auf ihren Status bedacht, ist sie nicht nur Objekt und Opfer ihres Hermann, sondern sie macht

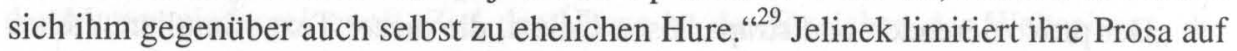
die sexuellen Akte, eliminiert bewusst die Beschreibungen danach oder davor. Sex gilt hier als Mittel, und nicht als Vergnügen. Wo ist denn Platz für Liebe? Das Buch lesend, kommt man zu der Überzeugung, Liebe habe mit Sex nichts zu tun. Die Beziehung von Gerti und Hermann weist in keinem Bereich dezente Gefühle und Empfindungen auf. Ihr Leben ist die Verwirklichung sexueller Männerphantasien, der ,männlichen Lust in der extremen Variante der alltäglichen häuslichen Vergewaltigung und der kommerziellen Gewalt-Pornographie" ${ }^{30}$ Beabsichtigt war es von Jelinek, einen Porno für Frauen zu schreiben, einen weiblichen Blick auf die Lust vorzustellen, sich mit der sexuellen Begierde der Frau zu befassen. „Sie sei gescheitert, nun schreibe sie einen Anti-Porno, in dem sie die männliche Sprache der Lust den Lesern ins Gesicht schlagen solle."31 "Gerti bleibt irregeleitet von den kulturell geprägten Erwartungsmustern von Liebe, wahrhaft künstlicher Paradiese, ein sexueller Gebrauchsgegenstand der Männer. “32

Wie in früheren Werken Jelineks wird auch in „Lust“ die Sexualität als ein Begriff der Herrschaft und Dominanz in der Beziehung verwendet. Zusätzlich knüpft Jelinek mit der Sexualität und ihrer drastischen Darstellung an die Medien und die durch sie verbreiteten Bilder vom Sex im ehelichen Leben an. „Die Macht der Me-

\footnotetext{
${ }^{25}$ Ebd., S. 3.

${ }^{26}$ Thomas Anz: Über die Lust und Unlust am Text, a. a. O., S. 3.

${ }^{27}$ Elfriede Jelinek: Lust, S. 150.

${ }^{28}$ Thomas Anz: Über die Lust und Unlust am Text., a. a. O., S. 1.

${ }^{29}$ Marlies Janz: Elfriede Jelinek, a. a. O., S. 112 f.

${ }^{30}$ Marlies Janz: Elfriede Jelinek, a. a. O., S. 111.

${ }^{31}$ Andreas Isenschmid, in: Elfriede Jelinek, a. a. O., S. 239.

${ }^{32}$ Klaus-Peter Philippi, in: Elfriede Jelinek. a. a. O., S. 237.
} 
dien und der audiovisuellen Porno-Industrie über das Alltagsverhalten ist das eigentliche Thema von Lust. ${ }^{33}$ Diese Meinung ist sehr radikal und weitblickend. Auch andere Motive werden von der Literaturkritik zu diesem Roman hervorgehoben. So werden die Medien und das Fernsehen nicht als Leitmotiv dieses Werkes gesehen. Die in Lust beschriebene klischeehafte Geschichte schildere eher das Problem von Sexismus: keine gleichen Rechte zwischen den Partnern. Bei der Analyse des Problems stößt man auf die Frage, warum sich Gerti nicht wehrt, warum sie nicht versucht, ihr Leben zu ändern? Man sieht zwar den misslungenen Versuch der neuen Chance mit Michael, aber muss auch dieser Versuch mit Sex enden? Die Frau scheint von ihrem eigenen Mann mit allen seinen Bedürfnissen, Möglichkeiten abhängig zu sein und geht mit ihrem Problem nicht nach draußen. Es fehlt hier an Kommunikation und Bewahrung des Selbstwertes. Ist sie sich überhaupt dessen bewusst, dass sie von dem Mann ausgenutzt sein wird? Jelinek bedient sich bei der Gestaltung ihrer Figuren ganz bewusst sehr markanter Persönlichkeiten. Als Gegenpole zwingt sie das Ehepaar zum Zusammenleben. Lediglich eine Nebenrolle spielt ihr einziges Kind, das von der Mutter getötet wird. Aus Frustration, Mangel an Selbstbewusstsein? Soll das möglicherweise der Protest gegen die Vermassung sein? „In der Aversion und im Verweigerungsgestus gegenüber einer durch und durch sexualisierten und genußfixierten Konsumgesellschaft und Kulturindustrie steht die Autorin von Lust den antihedonistischen Tendenzen von Adornos Kulturkritik nahe. Es gibt jedoch in der Perspektive Adornos (und wohl auch Jelineks) andere, höher bewertete Lüste, die sich von den Konsumgewohnheiten der Masse abheben: zum Beispiel die Lust am gesellschaftlich verdrängten Leid, am Negativen, am Dissonanten, die von hochwertigen Kunstwerken insofern ermöglicht wird, als sie die Erfahrung vermitteln, schlechten gesellschaftlichen Verhältnissen Widerstand entgegensetzen zu können. “34 Diese Provinz-Geschichte gehört unbestritten zu den wichtigsten Büchern von Jelinek. Sowohl Thema als auch spezielle sprachliche Mittel machen die Gesamtheit des Werkes aus. Durch die Komplexität der Beschreibungen, durch neue Wendungen und Wortschöpfungen nähern diese Prosa Jelineks an die Postmoderne an. Da hier alles zugelassen ist, wird das lineares Lesen erheblich erschwert. Der Text führt eigentlich zur „Einübung in die Mythendestruktion". 35

„Jelinek Werk kreise um Beziehungen zwischen den Geschlechtern und innerhalb der Familienbande, um Sexualität, Macht, Gewalt, Faschismus und Tourismus, irgendwie komme alles immer gleichzeitig vor mit leichten Akzentwechseln, sogar mal Ausflüge in die Philosophie, die Stücke, nun ja, skandalös oder auch geschmacklos inszeniert, man erinnerte an Skandale. ${ }^{\text {‘36 }}$

\footnotetext{
${ }^{33}$ Marlies Janz: Elfriede Jelinek, a. a. O., S. 112.

${ }^{34}$ Theodor Adorno: Ästhetische Theorie, in: Thomas Anz: Lust und Unlust am Text, S. 3.

${ }^{35}$ Marlies Janz: Elfriede Jelinek, a. a. O., S. 113.

${ }^{36}$ Andrea Geier: Lob mit Fußtritten. a. a. O., S. 2.
} 


\section{„Bukolit“}

„Die Elfriede Jelinek ist doch die Kassandra der zeitgenössischen Literatur und des deutschsprachigen Theaters. “37 Als drittes Buch von Jelinek, das früh entsteht, jedoch viel später erscheint, sei hier Bukolit angeführt. In diesem Werk findet sich einmal mehr die Bestätigung der Selbstbeschreibung der Autorin. „Das zornige junge Mädchen aus Österreich“38 ${ }^{\text {s3 }}$ schildert auch in diesem Hörspiel eigene Beobachtungen und seine Meinung über die sie umgebende Welt. Die Literatur wird hier als Ärgernis und Protest gegen die gesamte Welt verstanden und auch als Mittel der Weiterhandlung benutzt. Jelinek achtet hier sehr rigoros auf die Kleinschreibung, „das sind keine Mätzchen, sondern der konsequent betriebene Versuch, eine Tabula-rasa-Strategie für den gesellschaftlichen Bereich auch auf die Sprache auszudehnen. ${ }^{39}$ Die Demontage der Sprache in diesem Hörspiel führt zu einem Wörterstrom, der individuell vom Leser interpretiert werden kann. Die beiden Figuren bukolit und bukolita verschwinden in der „lockeren“ Konstruktion des Romans, in der Menge neuer Wörter und neuer Sinnzusammenhänge. Jelinek ,zielt darauf ab, literarische Konsumgewohnheiten zu verändern" ${ }^{40}{ }^{4}$ Das Chaos hat aber eine besondere Bedeutung und macht das Neuartige dieses Hörspiels in der damaligen Literatur aus. Die postmoderne Epoche und ihre Gewohnheiten, Neuschöpfungen inspirieren die Autorin. Es lässt sich nicht entscheiden, ob Jelinek in diesem Werk der Form oder dem Inhalt mehr Aufmerksamkeit schenkt. In den später entstandenen Werken sieht man ihre Konzentration auf den Inhalt. In Bukolit indes muss der Leser seine Phantasie bemühen und alle Lücken des Ungesagten selbständig ergänzen, um daraus ein logisches Ganzes zu bilden. Oder vielleicht war es gerade das Ziel von Jelinek, die Ganzheit nicht so einfach identifizierbar zu machen. Das Thema des Romans ist sehr Jelinek-typisch: Sex und Sexualität, allerdings in anderer Dimension und etwas anderer Bedeutung. Einerseits könnte man sich überlegen, ob das Hörspiel sich mehr mit einem frühreifen Mädchen befasst oder eher doch mit einer Sexmaschine? Der ganze Intimbereich der ganzen Publik steht doch enthüllt im Vordergrund. Und obwohl manche meinen, dieses Bild sei ganz grotesk dargestellt, verliert die Obszönität des Bildes nicht an Bedeutung. Die künstlerische Darstellung dieses Problems (oder nach Jelinek Gesellschaftserscheinung) scheint auch in diesem Werk ganz erfolgreich zu sein. Die Unmöglichkeit der klaren Identifikation des Helden und seiner vielen Rollen, in denen er sich vorstellt und die er annimmt, machen daraus den Eindruck eines viel mehr zusammengesetzten Romans. „Fetischismus und Heldenverehrung gibt die Autorin der Lächerlichkeit preis. In-

${ }^{37}$ Claus Peymann: Kommentar zum Nobelpreis für Jelinek, in: Andrea Geier: Lob mit Fußtritten, a. a. O., S. 3 .

\footnotetext{
${ }^{38}$ Hansjörg Graf, in: Elfriede Jelinek: Dossier, a. a. O., S. 190.

${ }^{39}$ Ebd., a. a. O., S. 190.

${ }^{40}$ Ebd., a. a. O., S. 190.
} 
dem sie Phrasen und Slogans freilegt, liquidiert sie Inhalte."41 Jelinek sieht ihre Welt nicht als Idealistin, sondern als Satirikerin. Die ganze Gesellschaft unterliegt einer eingehenden Analyse, und die Resultate werden dann grotesk und übertrieben im Werk dargestellt.

Bukolit gibt keine Antworten, die Welt der Frau und des Mannes scheint in diesem Werk nur weitere Fragen zu stellen. Fragen, auf die man keine Antwort kennt und keine erwartet. Fragen, die von selbst nicht beantwortet werden können und die in jedem Fall einer anderen Antwort bedürfen. Der Collageroman gilt hier als hervorragendes Beispiel der experimentellen Literatur. Die Spiele mit der Sprache, mit der Kleinschreibung stehen für die phantasievolle Darstellung der neuen Idee Jelineks. „Ein Wirrwarr von Bildern und Wörtern, von denen nur der aktive, assoziierende oder - und deshalb heißt der Roman Hörroman - der laut lesende Leser etwas hat." 42

Jelinek schafft in Bukolit eine Scheinwelt. Eine Welt, die allen bekannt ist, in der alle leben, die jedoch keiner akzeptiert. Die Scheinwelt ist ein Protest gegen die reale Welt. Der Roman ist Beispiel eines Protestes, einer Enthüllung des wahren Ichs. Die Gewohnheiten, die Rituale, das Gewöhnliche zerstören das geistige Leben. Es gibt hier kaum Raum für Psychologie und eine psychologische Analyse der Figuren. Ihr Leben, sehr krass und übertrieben dargestellt, ist zugleich eine Demonstration gegen die Langeweile des Alltags. Wortakrobatik und Phantasie sollen dem Leser die freie Interpretation des Stückes ermöglichen.

\section{Frauenliteratur - frauenregierte Welt}

„Mit Jelinek ist es gar eine Frau, die es wagt, Leser mit der Mächtigkeit (Potenz), Schärfe und Ironie ihrer Sprache zu konfrontieren - was im einzelnen Psychohaushalt vielleicht Unruhe stiftet - in der Gesamtheit der Empörten, sich gerne Empörenden aber selbstverständlich Konsens. “43 Verdrängte Sexualität, Entzug der Selbsterkenntnis, Abhängigkeit und direkte, bedingte Liebe zu einem Mann oder zur eigenen Mutter sind die am häufigsten anzutreffenden Themen in den Werken Jelineks. Die Figur einer Frau, die leidet, gequält wird oder alleine die eigene unbegrenzte Macht ausübt, lässt sich in jedem Werk wiederfinden. Gewaltzusammenhänge in den einzelnen Büchern bilden eine Kontinuität in Jelineks Schaffen. „Die entpolitisierende Jelinek-Rezeption, wie sie insgesamt vorherrscht, auch wo man pauschal ihre schwarzen Gesellschaftsbilder oder ihren - zumeist falsch verstanden - Feminismus goutiert, wäre dem Selbstverständnis der Autorin zufolge als Mythi-

\footnotetext{
${ }^{41}$ Ebd., S. 192.

${ }^{42}$ Hans Christian Kosler, in: Elfriede Jelinek: Dossier, a. a. O., S. 194.

${ }^{43}$ Elfriede Gerstl: Elfriede Jelinek, a. a. O., S. 1.
} 
sierungsprozess zu beschreiben. “44 Es werden aber auch andere Aspekte ihrer Literatur entdeckt: ,ihre Sensibilität für Mode, ihre Urbanität, ,Dandyhaftigkeit' in dem Sinn, dass Ästhetisches das bestimmende Prinzip aller Lebensbereiche ist. “45 „Das macht Jelinek zur exemplarischen Autorin der ästhetischen Moderne, einer Moderne, die in der deutschsprachigen Literatur, traut man nicht dem Schein, sondern der Sache, nie großen Anwert hatte, schon gar nicht heutzutage (...) ${ }^{\text {“46 }}$ „Darüber hinaus geht es bei Jelinek grundsätzlich nicht um das postmoderne Spiel mit kulturellen Mustern, sondern um deren satirische Entlarvung im Kontext von Feminismus und Faschismus-Kritik. “47 Die Literatur Jelineks ist eine experimentelle Literatur, in der sie mehrere Lücken findet und diese in der ihr eigenen Art füllt. „Von ihren systematischen Expeditionen über die Grenzen der Kunst hinaus brachte sie jene Schätze mit, die es ihr ermöglichten, nicht intellektuelle, sondern das Gegenteil: intelligente Literatur zu schreiben. “48 Vergebens sucht man bei Jelinek nach Idealbildern der Welt, des Menschen, einer Frau. Mit Radikalität, durch den Gebrauch von Politik und Ästhetik ruiniert sie bisherige Vorurteile, Ikonen des guten Benehmens. Das Bestehende wird von ihr in keiner Dimension akzeptiert. „Sie stellt in ihren Texten das Schlechte an den Zuständen dar, aber so, und darin ist Elfriede Jelinek unübertroffene Meisterin, dass das falsche, abgestorbene Leben noch einmal lebendig wird. Das Falsche vollkommen, geradezu in seiner Vollkommenheit zu erfassen, das ist vermutlich nicht nur heute das einzige, was sich über das Richtige, das möglich wäre, sagen und zeigen lässt. “"49

\section{Literatur}

Anz, Thomas: Über die Lust und Unlust am Text. Zu Elfriede Jelineks "Lust", in: C.H.Beck, Literaturkritik, Nr. 7, 2005.

Bartsch, Kurt, Höfler, Günther: Elfriede Jelinek Dossier, Literaturverlag Droschl, 1991.

Geier, Andrea: Lob mit Fußtritten. Über den Nobelpreis für Elfriede Jelinek, in: C.H.Beck, Literaturkritik, Nr.11, 2004.

Gerstl, Elfriede: „Schrillers weiss ich heute nicht anzubieten. “ Die Frau als Wutableiter in: Literaturlandschaft Österreich, 2003.

Janz, Marlies: Elfriede Jelinek, Metzler Verlag, Stuttgart 1995.

Jelinek, Elfriede: Bukolit, Rhombus Verlag, Wien 1979.

Jelinek, Elfriede: Die Ausgesperrten, Rowohlt Taschenbuch Verlag, Hamburg 1985.

Jelinek, Elfriede: Die endlose Unschuldigkeit, Schwiftinger Galerie Verlag, München 1980.

Jelinek, Elfriede: Lust, Rowohlt Taschenbuch Verlag, Hamburg 1992.

Jelinek, Elfriede: Pianistka, Wydawnictwo AB, Warszawa 2004.

Scharang, Michael: Elfriede Jelinek - ein Weltexperiment, in: Konkret. Heft 7, 2002.

\footnotetext{
${ }^{44}$ Marlies Janz: Elfriede Jelinek, a. a. O., S. VII.

${ }^{45}$ Elfriede Gerstl: Elfriede Jelinek, a. a. O., S. 2.

${ }^{46}$ Michael Scharang, Elfriede Jelinek - ein Weltexperiment, S. 1.

${ }^{47}$ Marlies Janz: Elfriede Jelinek, a. a. O., S. VIII.

${ }^{48}$ Michael Scharang, Elfriede Jelinek..., a. a. O., S. 1.

${ }^{49}$ Michael Scharang, Elfriede Jelinek - ein Weltexperiment, S. 2.
} 